\title{
Intravenous Immunoglobulin Therapy Restores the Quantity and Phenotype of Circulating Dendritic Cells and CD4+ T Cells in Children with Acute Kawasaki Disease
}

\section{Nana Wang}

Children's Hospital of Soochow University

\section{Zhongyue Chen}

Children's Hospital of Soochow University

\section{Fan Zhang}

Children's Hospital of Soochow University

\section{Qianwen Zhang}

Children's Hospital of Soochow University

\section{Ling Sun}

Children's Hospital of Soochow University

Haitao Lv

Children's Hospital of Soochow University

\section{Bo Wang}

Children's Hospital of Soochow University

Jie Shen

Children's Hospital of Soochow University

\section{Xufang Zhou}

Children's Hospital of Soochow University

\section{Feiyan Chen}

Children's Hospital of Soochow University

\section{Binwei Zhang}

Children's Hospital of Soochow University

\section{Lijun Meng}

Children's Hospital of Soochow University

\section{Huiting Zhou}

Pediatric Research Institute of Soochow University

\section{ZhenJiang Bai}

Children's Hospital of Soochow University

Jie Huang ( $\nabla$ j.shuang@163.com )

Children's Hospital of Soochow University 


\section{Research Article}

Keywords: Kawasaki disease, Intravenous immunoglobulin, Dendritic cells, CD4+ T cells

Posted Date: August 13th, 2021

DOI: https://doi.org/10.21203/rs.3.rs-798625/v1

License: (c) (1) This work is licensed under a Creative Commons Attribution 4.0 International License. Read Full License

Version of Record: A version of this preprint was published at Frontiers in Immunology on February 10th, 2022. See the published version at https://doi.org/10.3389/fimmu.2022.802690. 


\section{Abstract}

Background: Intravenous immunoglobulin (IVIG) is widely used to treat Kawasaki disease (KD). However, the mechanisms by which it reduces systemic inflammation are not completely understood. Dendritic cells (DCs) and T cells play critical roles in the pathogenic processes of immune disorders. Assessing the quantity of DC subsets and T cells and identifying functional molecules present on these cells, which provide information about KD, in the peripheral blood may provide new insights into the mechanisms of immunoglobulin therapy.

Methods: In total, 54 patients with KD and 27 age-matched healthy controls (HCs) were included in this study. The number, percentage, and phenotype of DC subsets and CD4 ${ }^{+} \mathrm{T}$ cells in peripheral blood were analysed through flow cytometry.

Results: Patients with KD exhibited fewer peripheral DC subsets and CD4 ${ }^{+} \mathrm{T}$ cells than HCs. Human leucocyte antigen-DR (HLA-DR) expression was reduced on myeloid DCs (CD1 $\mathrm{c}^{+} \mathrm{mDCs}$ ), whereas that on plasmacytoid DCs (pDCs) did not change significantly. Both pDCs and CD1 $\mathrm{c}^{+} \mathrm{mDC}$ displayed significantly reduced expression of co-stimulatory molecules, including CD40, CD86. Expression of T-cell immunoglobulin and mucin domain 3 (TIM-3) was increased on CD4 ${ }^{+}$T cells. No significant differences were observed concerning the quantity and phenotype of DC subsets and CD4 ${ }^{+} T$ cells in patients with KD with and without coronary artery lesions. Importantly, these altered quantity and phenotypes on DC subsets and $C D 4^{+} T$ cells were restored to a great extent after IVIG treatment. T helper (Th) subsets including Th1 and Th2 among CD $4^{+} \mathrm{T}$ cells did not show alteration pre- and post-IVIG treatment, although the Th1-related cytokine IFN-y level increased dramatically in patients with KD pre-IVIG treatment.

Conclusions: $\mathrm{pDC}$ and $\mathrm{CD} 1 \mathrm{c}^{+} \mathrm{DC}$ presented an immature or tolerant phenotype in acute stages of KD, IVIG treatment restored the quantity and functional molecules of DCs and $C D 4^{+} \mathrm{T}$ cells to distinct levels, indicating the involvement of $\mathrm{DCs}$ and $\mathrm{CD} 4^{+} \mathrm{T}$ cells in the inflammation in $\mathrm{KD}$. The findings provide insights into the immunomodulatory actions of IVIG.

\section{Introduction}

Kawasaki disease (KD) is a febrile systemic vasculitis that predominately affects children less than 5 years of age and is the leading cause of acquired cardiac disorders in children [1, 2]. Increasing evidence supports that immune-mediated inflammation plays an essential role in the pathophysiology of KD. However,to date, the mechanisms involved in the aetiology of KD have not been completely elucidated. Dendritic cells (DCs) are professional antigen presenting cells playing a key role in inducing the activation of naive $T$ cells and bridging innate and adaptive immunity [3-5]. DCs recognise the presence of pathogens through pattern recognition receptors, including TLRs, and further migrate from the periphery to the lymph nodes to present pathogen-derived antigens to T cells. Studies have indicated that DCs and T cells could play a key role in KD pathogenesis because mature and activated DCs and T cells expressing the HLA-DR antigen have been reported to infiltrate the coronary artery and skin lesions in 
patients with $\mathrm{KD}[6,7]$ and in the LCCWE-induced coronary artery lesion mouse model [8]. Altered activation and effector subsets (Th1 and Th2) have been reported but no consistent conclusion has been drawn yet.

Intravenous immunoglobulin (IVIG) treatment remains the most effective therapy for KD. Although the underlying mechanisms are not fully elucidated, it is well-accepted that IVIG cures patients by downregulating inflammatory responses, which further protects the vascular system and myocardium from immune-mediated damage. Diverse mechanisms have been suggested to explain the anti-inflammatory activity of IVIG, including the neutralisation of microbial toxins or cytokines [9], suppression of T- and Bcell activation [10], promotion of apoptosis of peripheral blood lymphocytes [11], regulation of Th17/Treg cell balance [12], and reduction in cytokine release [13, 14]. However, only a few studies have investigated the action of IVIG on DCs and CD4 ${ }^{+}$T cells in KD. Systemic studies on DCs and T cells are warranted to gain insights into the role of these immune cells in the KD pathogenesis and IVIG treatment.

In this study, we determined the frequency, number, and phenotype of peripheral pDCs and CD1 $\mathrm{c}^{+} \mathrm{mDCs}$. Moreover, we studied the expression of inhibitory receptors on $\mathrm{CD} 4^{+} \mathrm{T}$ cells and helper T-cell subsets in patients with KD and the changes in these parameters over the course of IVIG treatment.

\section{Materials And Methods Participants}

A total of 54 patients with KD admitted to the Department of Cardiology, Children's Hospital of Soochow University, from June 2020 to June 2021 participated in this study. All the participants conformed to the diagnostic criteria revised by AHA Kawasaki Disease Guideline [15]. All patients received intravenous immunoglobulin (IVIG,2 g/ $\mathrm{kg}$ ) in addition to oral aspirin $(50-80 \mathrm{mg} / \mathrm{kg} /$ day) as an initial treatment. Patients who fulfilled at least one of the following criteria were excluded: 1) having received corticosteroids and immunosuppressive drugs, 2) having received their initial IVIG infusion in other hospitals, 3) refused IVIG infusion, and 4) presented with recurrent KD. Echocardiography was performed in all the patients before IVIG treatment. Measurements of the diameter of the left main coronary artery, the anterior descending branch, the circumflex artery, and the right coronary artery were normalised based on the body surface area and expressed as a Z score (standard deviation units from the mean). A Z score $\geq 2$ was considered to indicate coronary artery lesion [16]. In total, 27 age-matched healthy controls who had received routine regular physical examinations were enrolled as healthy controls (HCs). Clinical parameters such as the white blood cell count (WBC), neutrophil count, lymphocyte count, $C$ reactive protein (CRP) level, and Prealbumin were recorded from both patients and HCs. The parents of all the study participants were informed about the study, and they provided informed consent.

\section{Surface phenotype characterisation with flow cytometer}


Peripheral blood $(2 \mathrm{~mL}$ ) was collected from $\mathrm{HCs}$ and patients with $\mathrm{KD}$ at two time points: before IVIG treatment (pre-IVIG) and 3 days after completing initial IVIG treatment (post-IVIG). Peripheral blood leukocytes were isolated from EDTA-blood through red blood cell (RBC) lysis within $4 \mathrm{~h}$. Briefly, RBCs were lysed using human erythrocyte lysing solution (BioLegend), and samples were washed twice with phosphate-buffered saline (PBS) without $\mathrm{Ca}^{2+}$ and $\mathrm{Mg}^{2+}$. After the samples were washed, peripheral blood leukocytes were resuspended in PBS containing 2.5\% foetal bovine serum (FBS) and incubated at $4^{\circ} \mathrm{C}$ for $30 \mathrm{~min}$ in dark with the following fluorochrome-conjugated monoclonal antibodies: CD3-FITC (SK7), CD14-FITC (HCD14), CD15-FITC (HI98), CD16-FITC (3G8), CD19-FITC (HIB19), CD20-FITC (2H7), CD56-FITC (MEM-188), HLA-DR-PE/CY7 (L243), CD123-APC(6H6), CD1C-PE(L161), CD86-PE/CY7(IT2.2), CD40-BV421(5C3), CD4-BV421 (RPA-T4), TIM-3-APC (F38-2F2), TIGIT-PE (A15153G), and PD-1-BV510 (NAT105). All the samples were washed and analysed using the Attune NxTflow cytometer (LifeTechnology). All antibodies were purchased from BioLegend (San Diego, CA, USA) or BD Biosciences (San Diego, CA, USA). Total DCs (pan-DCs) were characterised as negative for lineage markers (CD3, CD14, CD15, CD16, CD19, CD20, and CD56) and positive for HLA-DR (Fig. 1a). The myeloid

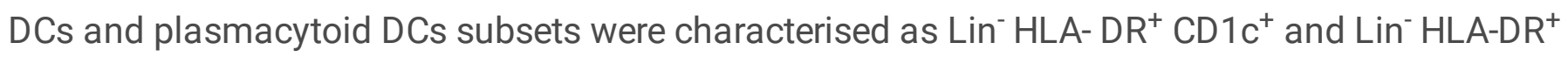
$\mathrm{CD}_{123^{+}}$, respectively (Fig. 1a). Dead cells were excluded through 7-aminoactinomycin D (7-AAD; BioLegend) viability staining. The absolute number of $\mathrm{CD} 1 \mathrm{c}^{+} \mathrm{mDCs}$ and $\mathrm{pDCs}$ was calculated from the peripheral blood leukocytes count multiplied by the proportion of each subset within peripheral blood leukocytes per millilitre, as determined by flow cytometric analysis.

\section{Intracellular cytokine determination of T cells}

For the detection of intracellular cytokines by flow cytometry, peripheral blood leukocytes were cultured in $10 \%$ foetal calf serum in RPMI- 1640 and incubated for $5 \mathrm{~h}$ at $37^{\circ} \mathrm{C}$ under $5 \% \mathrm{CO}_{2}$ in the presence of 5 $\mathrm{ng} / \mathrm{mL}$ phorbol myristate acetate (PMA), $0.5 \mu \mathrm{g} / \mathrm{mL}$ ionomycin, and Golgiplug (containing monensin, $1 / 1000$ final concentration). Cells were washed and stained with anti-CD 4 at $4^{\circ} \mathrm{C}$ for $30 \mathrm{~min}$ in the dark. After washed twice, cells were fixed with lysing buffer (BD Biosciences), permeabilized with permeabiliztion solution (BD Biosciences), and were then incubated with antibodies against IFN- $y$ and IL4 (BD Biosciences) for intracellular staining, according to the manufacturer's instructions. Th1 and Th2 cells were identified as $\mathrm{CD} 4^{+} \mathrm{IFN}-\mathrm{\gamma}^{+}$and $\mathrm{CD} 4^{+} \mathrm{IL}-4^{+}$, respectively. Dead cells were excluded using a fixable live/dead dye (Invitrogen). The data were analysed using FlowJo v10.4 software (FlowJo, OR, USA).

\section{Plasma cytokine measurement}

The Human Th1/Th2/Th17 Kit (BD Biosciences) was used to measure plasma cytokine levels. IL-2, IL-4, IL-6, IFN- $y$, TNF-a, IL-17A, and IL-10 levels were assessed. Data were analysed using CBA software. The individual cytokine concentrations were indicated by their fluorescence intensities. The concentrations of all the cytokines were reported in picogram per millilitre. 


\section{Statistical analysis}

Statistical analysis was performed using IBM SPSS Statistics 26.0. All data are expressed as median (interquartile range). For comparison between patients with KD and HCs, Mann-Whitney U-test was used. The Wilcoxon signed-rank test for paired samples was used for comparing the patients pre- and post-IVIG treatment. The significance of difference in sex distribution in patients and controls was analysed using the chi-square test. A $P$ value of $<0.05$ was considered significant.

\section{Results}

\section{Patient characteristics}

A total of 54 patients with KD and $27 \mathrm{HCs}$ were recruited based on our inclusion and exclusion criteria. Their characteristics are shown in Table 1. No significant differences in age and sex were observed between the groups. The WBC count, neutrophil count, prealbumin, and CRP levels were significantly higher in patients with KD pre-IVIG treatment than in $\mathrm{HCs}$, whereas no significant difference was observed in the absolute lymphocyte count. After IVIG treatment, WBC and neutrophil count and the CRP level decreased rapidly to an almost normal level, whereas the prealbumin level remained lower. According to echocardiography parameters, 52 patients with KD were divided into two groups: KD without coronary artery lesion (CALs; KD-NCAL) group $(n=40)$ and KD with CAL (KD-CAL) group $(n=14)$ (Table 2). No significant differences were observed in terms of WBC, neutrophil, and lymphocyte counts; neutrophil to lymphocyte ratio (NLR); and CRP and prealbumin levels between the KD-CAL and KD-NCAL groups.

Table 1

Characteristics of the study population. 


\begin{tabular}{|c|c|c|c|}
\hline \multirow[t]{2}{*}{ Parameters } & \multicolumn{2}{|l|}{ Kawasaki disease } & \multirow[t]{2}{*}{ Healthy controls } \\
\hline & Pre-IVIG & Post-IVIG & \\
\hline Number & 54 & 54 & 27 \\
\hline Age, months & $35.5(18.75-56.25)$ & $35.5(18.75-56.25)$ & $45(36-56)$ \\
\hline Sex, male/female & $29 / 25$ & $29 / 25$ & $16 / 11$ \\
\hline Fever duration before diagnosis & $5(4.0-6.25)$ & $5(4.0-6.25)$ & - \\
\hline WBC, $10^{9} / \mathrm{L}$ & $13.67(10.59-15.95)^{\mathrm{t, \neq}}$ & $7.54(5.41-10.47)$ & $7.38(6.16-8.33)$ \\
\hline Neutrophil, $10^{9} / \mathrm{L}$ & $8.21(6.09-11.36)^{\dagger, \ddagger}$ & $2.59(1.6-3.9)$ & $2.58(2.23-3.35)$ \\
\hline Lymphocytes, $10^{9} / \mathrm{L}$ & $3.21(2.52-4.12)$ & $3.38(2.66-4.88)$ & $3.68(2.89-4.08)$ \\
\hline $\mathrm{CRP}, \mathrm{mg} / \mathrm{L}$ & $49.04(34.42-85.57)^{t, \ddagger}$ & $5.9(3.12-11.86)^{\dagger}$ & $0.16(0.11-0.42)$ \\
\hline NLR & $2.52(1.79-3.88)^{\dagger, \ddagger}$ & $0.78(0.52-1.02)$ & $0.77(0.63-1.09)$ \\
\hline Prealbumin, mg/L & $75.5(58.0-87.25)^{t, \neq}$ & $166(137.75-200)^{\dagger}$ & $207(190-225)$ \\
\hline \multicolumn{4}{|c|}{$\begin{array}{l}\text { Baseline characteristics of the participants. Data shown are median (quartile spacing) or the number } \\
\text { of cases. -, this data was not detected; CRP, C-reactive protein; NLR, neutrophil to lymphocyte ratio; } \\
\text { WBC, white blood cell counts. } \\
{ }^{\dagger} P<0.05 \text { vs. the healthy controls. }{ }^{\ddagger} P<0.05 \text { vs. the post-IVIG treatment }\end{array}$} \\
\hline
\end{tabular}

Table 2

Characteristics of patients with KD classified according to coronary artery lesions or not. 


\begin{tabular}{|c|c|c|c|}
\hline \multirow[t]{2}{*}{ Parameters } & \multicolumn{2}{|l|}{ Kawasaki disease } & \multirow[t]{2}{*}{$P$ value } \\
\hline & NCAL & CAL & \\
\hline Number & 40 & 14 & \\
\hline Age, months & $33.5(20.0-50.0)$ & $45.4(14.5-66.75)$ & 0.4125 \\
\hline Sex, male/female & $22 / 18$ & $7 / 7$ & 0.7468 \\
\hline Fever duration before diagnosis & $6(4-6.75)$ & $4.5(4-6.25)$ & 0.2937 \\
\hline WBC, $10^{9} / \mathrm{L}$ & $13.67(10.14-15.97 \rrbracket$ & $13.96(11.43-15.77 \rrbracket$ & 0.5737 \\
\hline Neutrophil, $10^{9} / \mathrm{L}$ & $8.16(6.09-11.95 \rrbracket$ & 8.37(6.09-11.10区 & 0.8979 \\
\hline Lymphocytes, $10^{9} / \mathrm{L}$ & $3.17(2.53-4.11)$ & $3.32(2.19-4.30)$ & 0.6427 \\
\hline CRP, mg/L & $45.72(29.14-79.61)$ & $67.24(38.95-105.28)$ & 0.2690 \\
\hline NLR & $2.52(1.82-3.78)$ & $2.36(1.61-4.30)$ & 0.7596 \\
\hline Prealbumin, mg/L & $76(59.5-88)$ & $71.5(52.25-80.25)$ & 0.2645 \\
\hline
\end{tabular}

\section{Frequency and number of pDCs and CD1c ${ }^{+} \mathrm{mDCs}$}

To identify DC subsets, the gating strategy of total DCs and their subsets was shown (Fig. 1a). Quantitative flow cytometric analysis revealed that the frequency and absolute number of pDCs and $\mathrm{CD}_{1} \mathrm{c}^{+} \mathrm{mDCs}$ among pan-DCs significantly decreased in patients with KD pre-IVIG treatment compared with those in HCs $(P<0.0001$, Fig. 1b; $P<0.0001$, Fig. 1c; $P<0.0001$, Fig. 1 d; $P<0.0001$, Fig. 1e; respectively). Importantly, we found significantly recovered frequency and absolute number of pDCs and CD1c $\mathrm{c}^{+} \mathrm{mDC}$ in patients post-IVIG therapy $(P=0.0812$, Fig. 1b; $P=0.0006$, Fig. $1 \mathrm{c} ; P<0.0001$, Fig. $1 \mathrm{c}$; $P<$ 0.0001 , Fig. 1e; respectively). Although the percentage of both DC subsets in patients with KD did not recover to their levels observed in $\mathrm{HCs}(P<0.0001$, Fig. 1 b; $P<0.0001$, Fig. $1 \mathrm{~d}$; respectively), the absolute number of both DC subsets was same as that found in HCs. $(P=0.2255$, Fig. $1 \mathrm{c}$; $P=0.1730$, Fig. $1 \mathrm{e}$; respectively).

\section{Phenotypic properties on pDCs and CD1 ${ }^{+}$mDCs}

We compared the expressions of the antigen presenting molecule HLA-DR and co-stimulatory molecules (CD40 and CD86) in the two DC subsets in the peripheral circulation of patients with KD and HCs. These molecules are critical for DCs to elicit adaptive immune responses. 
Although no significant significance was observed between pre- and post-IVIG treatment in patients with $\mathrm{KD}$ and $\mathrm{HCs}$ in terms of pDCs, the MFI of the antigen presenting molecule HLA-DR exhibited a decreasing trend. ( $P=0.1822$, Fig. 2a; $P=0.3130$, Fig. 2a; respectively). CD40 percentage on pDCs in patients preIVIG treatment and MFI considerably decreased. ( $P<0.0001$, Fig. 2g; $P<0.0001$, Fig. 2h; respectively). IVIG treatment significantly elevated the percentage and MFI of CD40 expression on pDCs; however, they were not at normal levels as observed in $\mathrm{HCs}(P=0.0010$, Fig. $2 \mathrm{~g} ; P=0.0170$, Fig. $2 \mathrm{~h}$;

respectively). Similarly, the percentage and MFI of $C D 86^{+} \mathrm{pDCs}$ increased in patients post-IVIG treatment compared with that pre-IVIG treatment, with no difference between patients post-IVIG treatment and HCs. $(P=0.0016$, Fig. 2c; $P=0.0091$, Fig. 2d; $P=0.5288$, Fig. 2c; $P=0.5158$, Fig. 2d; respectively).

For $\mathrm{CD} 1 \mathrm{c}^{+} \mathrm{mDCs}$, we observed significantly decreased MFI of HLA-DR in patients with KD pre-treatment compared with that in $\mathrm{HCs}$, with no difference between post- treatment and $\mathrm{HCs}(P<0.0001$, Fig. $2 \mathrm{~b} ; P=$ 0.1368 , Fig. $2 \mathrm{~b}$; respectively). Unlike pDCs, the co-stimulatory molecule on $\mathrm{CD} 1 \mathrm{c}^{+} \mathrm{mDCs}$ in the patients was CD86 rather than CD40. A drastic decrease in the MFI and of $\mathrm{CD}^{+} 6^{+}$percentage was observed on CD1 $\mathrm{c}^{+} \mathrm{mDC}$ in the patients pre- IVIG treatment. $(P<0.0001$, Fig. 2e; $P<0.0001$, Fig. 2f; respectively). IVIG treatment significantly elevated the percentage and MFI of CD86 expression on CD1 $\mathrm{c}^{+} \mathrm{mDCs}$ in patients with $\mathrm{KD}$; however, these parameters were significantly lower than those in $\mathrm{HCs}(P<0.0001$, Fig. 2e; $P<$ 0.0001 , Fig. 2f; respectively). We did not observe obvious changes in terms of the percentage and MFI of CD40 on CD1c ${ }^{+}$mDCs between patients with KD and HCs $(P=0.6815$, Fig. 2i; $P=1609$, Fig. 2j; respectively).

Collectively, data presented in Fig. 1 indicated that the quantity of DCs and functional molecules on DCs were impaired in patients pre-IVIG treatment. IVIG treatment restored the quantity of DCs and functional molecules on DCs to distinct levels, indicating a possible role of DCs in the recovery of patients after IVIG treatment.

\section{Increased inhibitory receptor TIM-3 expression in patients with KD pre-IVIG treatment}

Considering the impaired quantity and functional molecules on DCs in patients with acute KD patients, we further assessed $C D 4^{+} T$ cells because $\mathrm{DCs}$ are the critical regulators of $\mathrm{CD} 4^{+} \mathrm{T}$ cells. The percentage and absolute number of $\mathrm{CD} 4^{+} \mathrm{T}$ cells decreased in the peripheral blood of patients pre-IVIG treatment compared with those in $\mathrm{HCs}(P<0.0001$, Fig. 3a; $P=0.0034$, Fig. 3b; respectively $)$. Subsequently, the percentage and number of $C D 4^{+} T$ cells increased after IVIG treatment, returning to normal levels $(P=$ 1027, Fig. 3a; $\mathrm{P}=08239$, Fig. 3b; respectively). Inhibitory receptors such as programmed cell death 1 (PD1), T cell immunoglobulin and ITIM domain (TIGIT), and T cell immunoglobulin and mucin domain 3 (TIM-3) are important molecules controlling T cell effector responses [17]. Inhibitory receptors have been involved in the pathophysiology of various human diseases, including autoimmune diseases $[17,18]$, sepsis [19], and cancer [20]. However, the expression of these inhibitory receptors on $\mathrm{CD}^{+} \mathrm{T}$ cells has not 
been reported previously in KD. To determine whether these inhibitory receptors are involved in the pathogenesis of KD『we used flow cytometry to assess the expressions of PD-1, TIGIT, and TIM-3 on peripheral $\mathrm{CD} 4^{+} \mathrm{T}$ cells. Representative flow cytometric analyses are shown in Fig. 3c, Patients with KD were found to exhibit a significantly higher expression of TIM-3 on $\mathrm{CD} 4^{+} \mathrm{T}$ cells pre-IVIG treatment than HCs, whereas no changes in PD-1 or TIGIT were observed between them. $(P<0.0001$, Fig. 3f; $P=0.2990$, Fig. 3d; $P=0.08123$, Fig. 3e; respectively). IVIG treatment significantly reduced percentage TIM-3 expression on $\mathrm{CD} 4^{+} \mathrm{T}$ cells, but not to a normal level as seen in HCs. $(P=0.0034$, Fig. $3 f)$. However, the percentage of $C D 4^{+}$T cells expressing the TIGIT was increased post-IVIG treatment $(P=0.0012$, Fig. 3e).

\section{Unaltered Th1/ Th2 polarisation of $\mathrm{CD}^{+} \mathrm{T}$ cells in patients with KD.}

Although many studies have focused on $T$ helper cytokines, most of them have determined the cytokines in plasma instead of $\mathrm{CD} 4^{+} \mathrm{T}$ cells. To understand the biology of $\mathrm{CD} 4^{+} \mathrm{T}$ cells, frequencies of different $\mathrm{CD} 4^{+} \mathrm{T}$-cell subsets were analysed based on cytokine patterns after in vitro stimulation of the T-cell receptor (TCR) signal. Production of intracellular IFN-y and IL-4, which are the representative factors of Th1 and Th2, respectively, in CD4 ${ }^{+} \mathrm{T}$ cells, was analysed in the peripheral blood of patients with $\mathrm{KD}$ and HCs. The gating strategy for determining Th1 and Th2 cells is shown in Fig. 4a. Neither the onset of KD nor IVIG treatment altered the percentages of Th1 and Th2 in CD $4^{+}$T cells. $(P=0.9851$, Fig. $4 \mathrm{~b} ; P=$ 0.7776 , Fig. 4b; $P=0.3980$, Fig. 4c; $P=0.5509$, Fig. 4c; respectively).

We further measured the level of a panel of Th subset-related cytokines in the plasma of patients with KD and $\mathrm{HCs}$. Of these, the plasma level of IL-4 was very low or undetectable in either study population (date not shown). IL-17A, IFN- $y$, TNF-a, IL-6, IL-10, and IL-2 levels were higher in patients with KD pre-IVIG than in HCs $(P=0.0464$, Fig. 4d; $P=0.0020$, Fig. 4e; $P=0.0084$, Fig. 4f; $P<0.0001$, Fig. 4g; $P<0.0001$, Fig. 4h; $P=0.0138$, Fig. 4i; respectively). These results are consistent with those previously reported [21, 22]. After IVIG treatment, plasma levels of IL-17A and IL-2 returned to normal $(P=0.2258$, Fig. $4 \mathrm{~d} ; P=0.1145$, Fig. 4i; respectively), IL-6 and IL-10 levels decreased, but levels were still significantly higher than in $\mathrm{HCs}(P<$ 0.0001 , Fig. 4g; $P=0.0056$, Fig. 4h; respectively). IFN-y and TNF-a levels had a tendency to be reduced, but levels were still significantly higher than in HCs. $(P=0.0122$, Fig. 4e; $P=0.0108$, Fig. 4f; respectively).

\section{Numbers and phenotypic properties on DC subsets and $\mathrm{CD}^{+} \mathrm{T}$ cells in patients with CAL and without CAL pre-IVIG treatment}

To investigate the correlation among DC subsets, $C D 4^{+} \mathrm{T}$ cells, and CAL, we performed a subgroup analysis comparing patients with and without CAL (KD-CAL and KD-NCAL groups). The number and proportion of circulating pDCs and $\mathrm{CD}_{1 \mathrm{c}^{+}} \mathrm{mDC}$ s were not significantly different in KD-CAL and KD-NCAL 
groups (Table 3). The expressions of HLA-DR, CD86, and CD40 on pDCs and CD1 $\mathrm{c}^{+} \mathrm{mDC}$ did not differ significantly between KD-CAL and KD-NCAL groups (Table 4). Furthermore, no difference was observed in the number, proportion, and TIM-3 receptor expression on $\mathrm{CD} 4^{+} \mathrm{T}$ cells between the two groups (Table 5).

Table 3

Percentage and number of DC subsets between KD patients with and without CAL.

\begin{tabular}{|llll|}
\hline Parameters & \multicolumn{2}{l|}{ Kawasaki disease } & Pvalue \\
\cline { 2 - 3 } & NCAL $(\mathrm{n}=40)$ & $\mathrm{CAL}(\mathrm{n}=14)$ & \\
\hline $\mathrm{pDCs} \%$ & $18.45(11.82-32.28)$ & $31.15(14.24-41.48)$ & 0.1671 \\
\hline $\mathrm{pDCs}(/ \mathrm{ml})$ & $4964(2979-7212)$ & $10425(4024-24450)$ & 0.0725 \\
\hline $\mathrm{CD} 1 \mathrm{c}+\mathrm{mDCs} \%$ & $7.84(4.72-14.93)$ & $8.44(5.69-15.28)$ & 0.8668 \\
\hline $\mathrm{CD} 1 \mathrm{c}+\mathrm{mDCs}(/ \mathrm{ml})$ & $2440(900-4846)$ & $3146(1688-4937)$ & 0.6498 \\
\hline $\begin{array}{l}\text { Data shown are median (quartile spacing) or the number of cases. CAL, coronary artery lesion; NCAL, } \\
\text { without coronary artery lesion. }\end{array}$ & \\
\hline
\end{tabular}

Table 4

HLA-DR, CD86 and CD40 expression on DC subsets between KD patients with and without CAL. 


\begin{tabular}{|c|c|c|c|}
\hline \multirow[t]{2}{*}{ Parameters } & \multicolumn{2}{|l|}{ Kawasaki disease } & \multirow[t]{2}{*}{$P$ value } \\
\hline & NCAL $(n=29)$ & CAL $(n=13)$ & \\
\hline MFI of HLA-DR on pDCs & 11826 (8605-15739) & 16625 (13268-18988) & 0.0586 \\
\hline MFI of HLA-DR on $\mathrm{CD}_{1} \mathrm{c}^{+} \mathrm{mDCs}$ & $16360(13777-21717)$ & $18561(14376-22444)$ & 0.5586 \\
\hline $\mathrm{CD} 86^{+}$expression on $\mathrm{pDCs} \%$ & $22.6(15.8-27.2)$ & $20.5(15.25-28.0)$ & 0.7543 \\
\hline $\mathrm{CD} 6^{+}$expression on $\mathrm{CD} 1 \mathrm{c}^{+} \mathrm{mDCs} \%$ & $47.8(38.56-56.2)$ & $47.7(34.4-60.15)$ & 0.6534 \\
\hline MFI of CD86 on pDCs & $224(142-294)$ & $204(149-242)$ & 0.6147 \\
\hline MFI of $\mathrm{CD} 86$ on $\mathrm{CD}_{1} \mathrm{c}^{+} \mathrm{mDCs}$ & $915(850-1145)$ & $967(738-1102)$ & 0.3914 \\
\hline $\mathrm{CD} 40^{+}$expression on $\mathrm{pDCs} \%$ & $4.20(2.05-7.61)$ & $6.07(3.41-11.65)$ & 0.0866 \\
\hline $\mathrm{CD}_{40} 0^{+}$expression on $\mathrm{CD} 1 \mathrm{c}^{+} \mathrm{mDCs} \%$ & $8.29(6.21-17.65)$ & $6.45(5.21-12.1)$ & 0.1310 \\
\hline MFI of CD40 on pDCs & $274(239-404)$ & $341(274-430)$ & 0.0997 \\
\hline $\mathrm{MFI}$ of $\mathrm{CD} 40$ on $\mathrm{CD} 1 \mathrm{c}^{+} \mathrm{mDCs}$ & $430(318-1007)$ & $304(265-577)$ & 0.0643 \\
\hline
\end{tabular}

Table 5

$\mathrm{CD} 4^{+}$and $\mathrm{TIM}-3^{+} \mathrm{CD} 4^{+} \mathrm{T}$ cells between KD patients with and without CAL.

\begin{tabular}{|c|c|c|c|}
\hline \multirow[t]{2}{*}{ Parameters } & \multicolumn{2}{|l|}{ Kawasaki disease } & \multirow[t]{2}{*}{$P$ value } \\
\hline & $\operatorname{NCAL}(n=25)$ & $\operatorname{CAL}(n=9)$ & \\
\hline $\mathrm{CD} 4^{+} \mathrm{T}$ cells $\%$ & $6.47(4.68-9.71)$ & $3.83(2.30-7.29)$ & 0.0859 \\
\hline \multirow[t]{2}{*}{$\mathrm{CD}^{+} \mathrm{T}$ cells $(/ \mathrm{ml})$} & 42034115 & 41448706 & 0.9842 \\
\hline & $(24620739-62163389)$ & $(24919683-74148861)$ & \\
\hline TIM- $3^{+}$expression on $\mathrm{CD} 4^{+} \mathrm{T}$ cells $\%$ & $5.58(4.20-7.81)$ & $6.93(4.92-8.73)$ & 0.2717 \\
\hline
\end{tabular}

\section{Discussion}


In this study, we report that the frequency and number of $\mathrm{pDCs}$ and $C D 1 \mathrm{c}^{+} \mathrm{DCs}$ and the expression of the antigen presenting molecule HLA-DR and co-stimulatory molecules, CD86 and/or CD40, on DCs decrease in acute stages of KD. IVIG treatment restored the quantity of DCs and functional molecules on DCs to distinct levels. In addition, our data indicated that the frequency and number of $\mathrm{CD} 4^{+} \mathrm{T}$ cells decreased in peripheral blood leukocytes in patients pre-IVIG treatment and were restored to a normal level after IVIG treatment. The expression of the inhibitory receptor TIM-3 on peripheral $\mathrm{CD} 4^{+} \mathrm{T}$ cells increased in patients pre-IVIG treatment and decreased post- IVIG treatment but not to a normal level. Although the levels of characteristic inflammatory cytokines including IL-2, IL-6, IFN- - , TNF-a, IL-17A, and IL-10 in patients with acute KD increased significantly compared with those in $\mathrm{HCs}$, we did not observe any changes in Th subsets when gated on peripheral $\mathrm{CD} 4^{+} \mathrm{T}$ cells. No significant differences were observed concerning the quantity and phenotype of DC subsets and $C D 4^{+} T$ cells between the KD-CAL and KD-NCAL groups.

For DC subsets, contradictory results have been reported in humans regarding numerical abnormalities of peripheral blood DC subtypes in KD [23, 24]. Suda et al. reported a decreased number of circulating mDCs but not of pDCs [24]. Burns et al. reported an increase in circulating mDCs but not in pDCs in the acute phase of KD [23]. Such contradictory results may be related to the different markers used for DC classification because DCs were identified through Lin $^{-}$(CD3, CD14, CD15, CD16, CD19, CD20, CD56) and HLA-DR staining in our study, the makers of DC staining are consistent with those of previous report [25]. However, Suda et al. identified DCs as Lin- (CD3, CD14, CD16, CD19, CD20, CD56) and HLA-DR [24]. Burns et al. relied upon CD11b and CD11c markers to identify DCs [23]. Reduction in the number of DCs may be because of alterations in DC viability, impaired differentiation with progenitor cells, or altered tissue distribution under inflammation, although the precise mechanism remains to be further validated. Studies have suggested that DCs migrate from the periphery to the site of coronary artery injury, given that mature and activated DCs infiltrate and accumulate around the coronary arteries. In conjunction with alterations in the percentage and absolute number of DC subsets, markers expressed on the surface of DCs that reflect their function are also significantly altered in KD. Expression of HLA-DR, a professional antigen presenting molecule, decreased significantly on $\mathrm{CD} 1 \mathrm{c}^{+} \mathrm{mDC}$ in $\mathrm{KD}$. We observed downregulated expressions of CD40 and CD86 on pDCs in patients with KD compared with those in HCs. Similarly, $\mathrm{CD}_{1 \mathrm{c}^{+}} \mathrm{mDC}$ also exhibited significantly lower levels of CD86; however, the expression of CD40 did not differ compared with that in HCs. Studies have established that mature DCs display a phenotype characterised by high surface expression of antigen presenting molecules and co-stimulatory molecules such as CD86 and CD40 [26, 27]. Immature DCs are known to have a lower potential to activate T cells [28]. Our results indicated that the circulating $\mathrm{PDCs}$ and $\mathrm{CD} 1 \mathrm{c}^{+} \mathrm{mDC}$ in patients with $\mathrm{KD}$ might reflect a tolerant or less mature phenotype in this condition. Importantly, studies on humans and animal models have indicated the presence of mature and activated DCs in CALs [6], which is probably due to an increased efflux of more mature DCs into the affected coronary artery and/or an increased influx of less mature DCs from the bone marrow, However, the assumption requires further validation.

The patients in our study exhibited a profoundly decreased percentage and number of $\mathrm{CD} 4^{+} \mathrm{T}$ cells, consistent with a previous report by Lee et al. [29] Numerous immunological studies on peripheral blood 
lymphocytes have been conducted, however, the role of T cells and the functional state of Th1 and Th2 cells remains controversial. Matsubara et al. reported a decrease in the number of Th1 type $\mathrm{CD}^{+} \mathrm{T}$ cell in the peripheral blood of patients with acute stage of KD and suggested the presence of a Th1/Th2 imbalance, particularly Th2 dominance [30]. Lee et al. reported that both Th1 and Th2 cells may be activated simultaneously during the acute stage of KD [22], whereas Kimura et al. suggested that the production of Th1 and Th2 cytokines is suppressed at the level of transcriptional regulation in KD [31]. Interestingly, we did not observe any change in Th subsets among $C D 4^{+} \mathrm{T}$ cells between patients with $\mathrm{KD}$ and $\mathrm{HCs}$. Lee et al. determined the cytokines in plasma instead of $\mathrm{CD} 4^{+} \mathrm{T}$ cells [22]. Kimura et al. analysed mRNA levels of T-bet and GATA-3, along with those of IFN- $y$ and IL-4, in peripheral blood mononuclear cells but not in CD4 ${ }^{+} \mathrm{T}$ cells [31]. Th1 (IFN-y-producing CD4 ${ }^{+} \mathrm{T}$ cells) and Th2 (IL-4producing $\mathrm{CD} 4^{+} \mathrm{T}$ cells) cells were identified by intracellular cytokine staining in our study. These experimental differences, as well as other methodological differences may account for the discrepancy. The function of T cells is regulated by inhibitory receptors such as PD-1, TIGIT, and TIM-3[17]. In this regard, none of the studies have reported the expression of PD-1, TIGIT, and TIM-3 expression in human $\mathrm{CD} 4^{+} \mathrm{T}$ cells. Augmented TIM-3 expression was observed on $\mathrm{CD} 4^{+} \mathrm{T}$ cells, whereas no changes in PD-1 and TIGIT expressions were observed in patients with KD pre-IVIG treatment with those in HCs. Given that TIM-3 is a negative regulatory molecule on $\mathrm{CD} 4^{+} \mathrm{T}$ cells, patients with $\mathrm{KD}$ might exhibit $\mathrm{CD} 4^{+} \mathrm{T}$ cells in a more suppressed state than HCs.

However, no significant differences were found concerning the quantity and phenotype of DC subsets and $\mathrm{CD}^{+} \mathrm{T}$ cells in patients with and without CAL in the acute phase of KD. Studies have reported the associations of CALs with several clinical variables, particularly CRP and NLR. In our clinical data, patients with CAL exhibited no significant elevation in CRP and NLR. In addition, not all of the patients with high CRP and NLR had CALs. The reason for these discrepant results is unclear; however, it may be related to various factors including different genetic backgrounds [32], pathogen species [33], and epigenetic effects [34].

Overall, these results strongly implicated that $\mathrm{pDCs}, \mathrm{CD} 1 \mathrm{c}^{+} \mathrm{mDCs}, \mathrm{CD} 4^{+} \mathrm{T}$ cells are in a suppressed state in the acute phase of KD. Our results are not consistent with the findings of Burns et al. [23], who reported an increased number of mDCs expressing CD86 in the acute KD phase. This difference might be because of the inclusion of CD14-positive cells, since monocytes have CD14 on their cell surface, whereas mDCs generally lack CD14. It remains uncertain whether peripheral blood T cells are activated in acute KD phases. The increased expression of TIM-3 on $\mathrm{CD} 4^{+} \mathrm{T}$ cells as observed in patients with KD may be associated with the suppression of the immune response. Our results are consistent with those of a study by Kuijpers et al. [35], who reported that a dysregulated TcR/CD3-dependent T cell unresponsiveness in acute KD. Additionally, Matsubara et al. suggested that T cells in the peripheral blood of patients with KD were not activated because of the low expression levels of intracellular CTLA-4 [36]. Ikeda et al. performed the microarray analysis of peripheral blood mononuclear cells and reported that the expressions of genes involved in antigen processing and presentation, the TCR signalling pathway, and the B-cell receptor signalling pathway were downregulated in acute phase KD [37]. Thus, the decline in the 
number of $\mathrm{pDCs}$ and $\mathrm{CD} 1 \mathrm{c}^{+} \mathrm{mDC}$ in patients with $\mathrm{KD}$ and their immature phenotype and the increase in TIM-3 expression on CD4 ${ }^{+} \mathrm{T}$ cells suggested a deficiency in the defensive system of patients with $\mathrm{KD}$ and may account for a high infection rate in these patients.

The mechanisms of action of IVIG in KD have been studied extensively; however, the role of DCs and $\mathrm{CD} 4^{+} \mathrm{T}$ cells in the resolution of inflammation in response to IVIG treatment has rarely been investigated. A study demonstrated the stimulation of normal donor tolerogenic mDCs in vitro, with Fc-induced IL-10 secretion, which influenced the differentiation of $T$ cells to regulatory $T$ cells; this finding indicated that the protective effects of IVIG treatment were, at least, partly mediated by Fc [23]. In this study, the number of circulating pDCs and CD1 $\mathrm{c}^{+} \mathrm{mDC}$ s increased to the normal levels after IVIG treatment. Moreover, IVIG treatment restored the expression of HLA-DR, CD86, and CD40 on DC subsets to different degrees. In addition, the $\mathrm{CD} 4^{+} \mathrm{T}$ cells were affected by IVIG treatment. The percentage of $\mathrm{CD} 4^{+} \mathrm{T}$ cells expressing the inhibitory receptor TIM-3 was decreased after IVIG treatment. To the best of our knowledge, this study is the first to describe the variation in inhibitory receptor expression on $\mathrm{CD} 4^{+} \mathrm{T}$ cells in KD. These results demonstrated that IVIG induced multiple phenotypic and functional changes in DC subsets and CD $4^{+} \mathrm{T}$ cells, mainly by promoting the resolution of inflammation, in patients with KD. The specific mechanisms leading to these effects should be further investigated.

Our study has some limitations. First, this preliminary study included a relatively small cohort of patients. Large-scale, multi-centre prospective studies are needed to confirm our findings. Second, this study involved short-term IVIG treatment; therefore, additional studies are required to elucidate the effects of IVIG on DC subsets and T cells throughout the entire inflammatory phase. Third, a further analysis of the function of these circulating DCs in patients with KD was hampered by a small number of DCs in the peripheral circulation. Hence, to confirm the functional characteristics of DCs and CD4 ${ }^{+} T$ cells, further in vitro and animal studies are needed. Fourth, we could not analyse the relationship between IVIGnonresponsive and $\mathrm{DC}$ subsets and $\mathrm{CD} 4^{+} \mathrm{T}$ cells because our study did not incorporate this study group. These issues will be addressed in our subsequent studies.

\section{Conclusions}

Collectively, our data indicated that IVIG could restore the percentage, number, and expression of effector molecules in DC subsets. Moreover, the expression of TIM-3 on CD4 ${ }^{+} \mathrm{T}$ decreased after IVIG treatment, suggesting that IVIG played a role in inhibiting TIM-3 expression, which might be one of the mechanisms of action of IVIG in KD treatment. Changes in DC subsets and CD4 ${ }^{+} \mathrm{T}$ cells contributed to the restoration of the immune balance, which can be used as an indicator of clinical monitoring of the immune status in patients with KD. This study offers us an insight into KD pathogenesis and provides a new perspective for understanding the mechanisms of action of IVIG.

\section{Abbreviations}


CBA: Cytometric bead array; DCs: Dendritic cells; HCs: Healthy controls; FBS: foetal bovine serum HLA-DR: Human leucocyte antigen-DR; IVIG: intravenous immunoglobulin; IL: Interleukin; IFN-ץ: Interferon- $\gamma ;$ KD: Kawasaki disease; mDCs: myeloid dendritic cells; pDCs: plasmacytoid dendritic cells; MFI: Mean fluorescence intensity; NLR: neutrophil to lymphocyte ratio; PD-1: Programmed cell death 1; PMA: phorbol myristate acetate; TIM-3: T-cell immunoglobulin and mucin domain 3; Th: T helper; TIGIT: T-cell immunoglobulin and ITIM domain; TNF-a: Tumor necrosis factor-a; 7-AAD: 7-aminoactinomycin D;

\section{Declarations}

\section{Acknowledgments}

The authors thank the volunteers for participation in this study.

\section{Authors' contributions}

$\mathrm{JH}$ and LM designed and supervised the study and revised the manuscript. $\mathrm{FZ}, \mathrm{QZ}, \mathrm{XZ}, \mathrm{FC}$, and $\mathrm{BZ}$ collected clinical information and samples. LM and NW wrote the manuscript. NW, ZC carried out the experiments, and analyzed, and interpreted the data. LM, ZB,HZ, LS, HL, BW, and JS interpreted the data and discussed the results, which are vital for the formation of conception. All authors participated in the manuscript review. The authors read and approved the final manuscript.

\section{Funding}

This work is supported by the National Natural Science Foundation of China (31670853), the Natural Science Foundation of Jiangsu Province (BK20190053), the Medical Science Program of Jiangsu Province (H2019002), the Talent Engineering Project of Jiangsu Province (BRA2018393), the Suzhou Clinical Medicine Expert Project (GSWS2019015), the Science and Technology Program of Suzhou (SYS2018067), and the Postgraduate Research \&Practice Innovation Program of Jiangsu Province (KYCX20_2725).

\section{Availability of data and materials}

All data used and analyzed during the current study are available from the corresponding author on reasonable request.

\section{Ethics approval and consent to participate.}

The experimental protocols were established following the Declaration of Helsinki and approved by the Ethics Committee of Children's Hospital of Soochow University. Written informed consent was obtained 


\section{Consent for publication}

Not applicable.

\section{Competing interests}

The authors declare that they have no competing interests.

\section{Author details}

${ }^{1}$ Department of Cardiology, Children's Hospital of Soochow University, Suzhou 215025, China.

${ }^{2}$ Department of Hematology, Children's Hospital of Soochow University, Suzhou 215025, China. ${ }^{3}$ Pediatric Research Institute of Soochow University, Suzhou 215025, China. ${ }^{4}$ Pediatric Intensive Care Unit, Children's Hospital of Soochow University, Suzhou 215025, China.

\section{References}

1. Singh S, Vignesh P, Burgner D, et al. The epidemiology of Kawasaki disease: a global update. Arch Dis Child. 2015;100(11):1084-8.

2. Wood LE, Tulloh R, et al. Kawasaki disease in children. Current Opinion in Rheumatology. 2009;10(10):787-92.

3. Steinman RM, et al. Decisions about dendritic cells: past, present, and future. Annu Rev Immunol. 2012;30(1-22.

4. O'Keeffe M, Mok WH, Radford KJ, et al. Human dendritic cell subsets and function in health and disease. Cell Mol Life Sci. 2015;72(22):4309-25.

5. Rossi M, Young JW, et al. Human dendritic cells: potent antigen-presenting cells at the crossroads of innate and adaptive immunity. J Immunol. 2005;175(3):1373-81.

6. Yilmaz A, Rowley A, Schulte D, et al., et al. Activated myeloid dendritic cells accumulate and colocalize with $\mathrm{CD}^{+} \mathrm{T}$ cells in coronary artery lesions in patients with Kawasaki disease. Experimental and molecular pathology. 2007;83(1):93-103.

7. Sato N, Sagawa K, Sasaguri Y, Inoue O, Kato H, et al. Immunopathology and cytokine detection in the skin lesions of patients with Kawasaki disease. The Journal of pediatrics. 1993;122(2):198-203.

8. Schulte DJ, Yilmaz A, Shimada K, et al., et al. Involvement of innate and adaptive immunity in a murine model of coronary arteritis mimicking Kawasaki disease. J Immunol. 2009;183(8):5311-8.

9. Shimozato T, Iwata M, Tamura N, et al. Suppression of tumor necrosis factor alpha production by a human immunoglobulin preparation for intravenous use. Infection and immunity. 1990;58(5):1384- 
90.

10. Leung D, Burns J, Newburger J, Geha R, et al. Reversal of lymphocyte activation in vivo in the Kawasaki syndrome by intravenous gammaglobulin. The Journal of clinical investigation. 1987;79(2):468-72.

11. Yi Q, Li C, Yang X, et al. Effect of intravenous immunoglobulin on inhibiting peripheral blood lymphocyte apoptosis in acute Kawasaki disease. Acta paediatrica (Oslo, Norway: 1992). 2001;90(6):623-7.

12. Guo M, Tseng W, Ko C, et al., et al. Th17- and Treg-related cytokine and mRNA expression are associated with acute and resolving Kawasaki disease. Allergy. 2015;70(3):310-8.

13. Ephrem A, Chamat S, Miquel C, et al., et al. Expansion of CD4 + CD25 + regulatory T cells by intravenous immunoglobulin: a critical factor in controlling experimental autoimmune encephalomyelitis. Blood. 2008;111(2):715-22.

14. Altara R, Mallat Z, Booz G, Zouein F, et al. The CXCL10/CXCR3 Axis and Cardiac Inflammation: Implications for Immunotherapy to Treat Infectious and Noninfectious Diseases of the Heart. Journal of immunology research. 2016;2016(4396368.

15. McCrindle BW, Rowley AH, Newburger JW, et al., et al. Diagnosis, Treatment, and Long-Term Management of Kawasaki Disease: A Scientific Statement for Health Professionals From the American Heart Association. Circulation. 2017;135(17):e927-e99.

16. Dallaire F, Dahdah N, et al. New equations and a critical appraisal of coronary artery Z scores in healthy children. J Am Soc Echocardiogr. 2011;24(1):60-74.

17. Anderson A, Joller N, Kuchroo V, et al. Lag-3, Tim-3, and TIGIT: Co-inhibitory Receptors with Specialized Functions in Immune Regulation. Immunity. 2016;44(5):989-1004.

18. Wang S, Zhang X, Leng S, et al., et al. Immune Checkpoint-Related Gene Polymorphisms Are Associated With Primary Immune Thrombocytopenia. Frontiers in immunology. 2020;11(615941.

19. McBride M, Patil T, Bohannon J, et al., et al. Immune Checkpoints: Novel Therapeutic Targets to Attenuate Sepsis-Induced Immunosuppression. Frontiers in immunology. 2020;11(624272.

20. von Witzleben A, Fehn A, Grages A, et al., et al. Prospective longitudinal study of immune checkpoint molecule (ICM) expression in immune cell subsets during curative conventional therapy of head and neck squamous cell carcinoma (HNSCC). International journal of cancer. 2021;148(8):2023-35.

21. Guo MM, Tseng WN, Ko CH, et al., et al. Th17- and Treg-related cytokine and mRNA expression are associated with acute and resolving Kawasaki disease. Allergy. 2015;70(3):310-8.

22. Lee S, Kim Y, Hyun M, et al., et al. T-Helper Cytokine Profiles in Patients with Kawasaki Disease. Korean circulation journal. 2015;45(6):516-21.

23. Burns JC, Song Y, Bujold M, et al., et al. Immune-monitoring in Kawasaki disease patients treated with infliximab and intravenous immunoglobulin. Clin Exp Immunol. 2013;174(3):337-44.

24. Tomoyuki Takahashi SK, et al. Circulating Myeloid Dendritic Cells is Decreased in the Acute Phase of Kawasaki Disease. Journal of Clinical \& Experimental Cardiology. 2013;04(10): 
25. Meng L, Bai Z, He S, et al., et al. The Notch Ligand DLL4 Defines a Capability of Human Dendritic Cells in Regulating Th1 and Th17 Differentiation. Journal of immunology (Baltimore, Md: 1950). 2016;196(3):1070-80.

26. Sousa C, et al. Reis e Sousa. Dendritic cells in a mature age. Nat Rev Immunol 6: 476-483. Nature reviews Immunology. 2006;6(6):476 - 83.

27. Tan JK, O'Neill HC, et al. Maturation requirements for dendritic cells in T cell stimulation leading to tolerance versus immunity. J Leukoc Biol. 2005;78(2):319-24.

28. Ganguly D, Haak S, Sisirak V, Reizis B, et al. The role of dendritic cells in autoimmunity. Nat Rev Immunol. 2013;13(8):566-77.

29. Lee H, Kim D, Noh G, Lee K, et al. Effects of intravenous immune globulin on the peripheral lymphocyte phenotypes in Kawasaki disease. Yonsei medical journal. 1996;37(5):357-63.

30. Matsubara T, Katayama K, Matsuoka T, et al., et al. Decreased interferon-gamma (IFN-gamma)producing $T$ cells in patients with acute Kawasaki disease. Clinical and experimental immunology. 1999;116(3):554-7.

31. Kimura J, Takada H, Nomura A, et al., et al. Th1 and Th2 cytokine production is suppressed at the level of transcriptional regulation in Kawasaki disease. Clinical and experimental immunology. 2004;137(2):444-9.

32. Kuo H, Li S, Guo M, et al., et al. Genome-Wide Association Study Identifies Novel Susceptibility Genes Associated with Coronary Artery Aneurysm Formation in Kawasaki Disease. PloS one. 2016;11(5):e0154943.

33. Tahara M, Baba K, Waki K, Arakaki Y, et al. Analysis of Kawasaki disease showing elevated antibody titres of Yersinia pseudotuberculosis. Acta paediatrica (Oslo, Norway: 1992). 2006;95(12):1661-4.

34. Kuo H, Li S, Huang L, Huang Y, et al. Epigenetic hypomethylation and upregulation of matrix metalloproteinase 9 in Kawasaki disease. Oncotarget. 2017;8(37):60875-91.

35. Kuijpers T, Wiegman A, van Lier R, et al., et al. Kawasaki disease: a maturational defect in immune responsiveness. The Journal of infectious diseases. 1999;180(6):1869-77.

36. Matsubara T, Anwar R, Fujiwara M, Ichiyama T, Furukawa S, et al. CTLA-4 (CD152) expression in peripheral blood T cells in Kawasaki disease. Clinical and experimental immunology. 2003;132(1):169-73.

37. Ikeda K, Yamaguchi K, Tanaka T, et al., et al. Unique activation status of peripheral blood mononuclear cells at acute phase of Kawasaki disease. Clinical and experimental immunology. 2010;160(2):246-55.

\section{Figures}


a
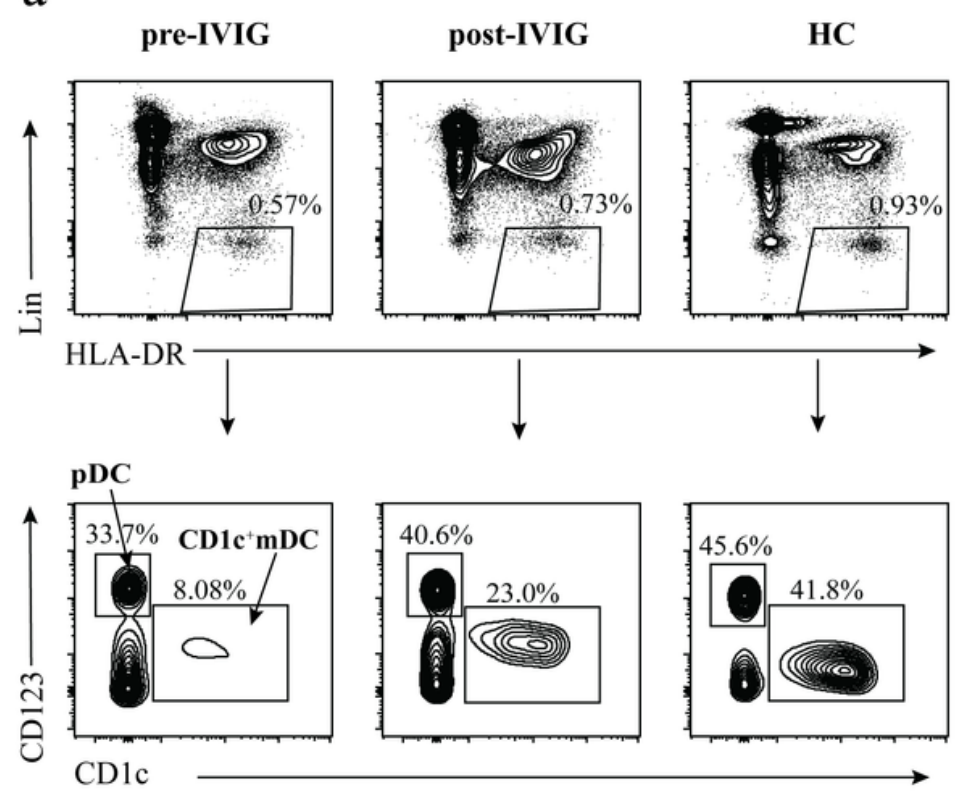

b
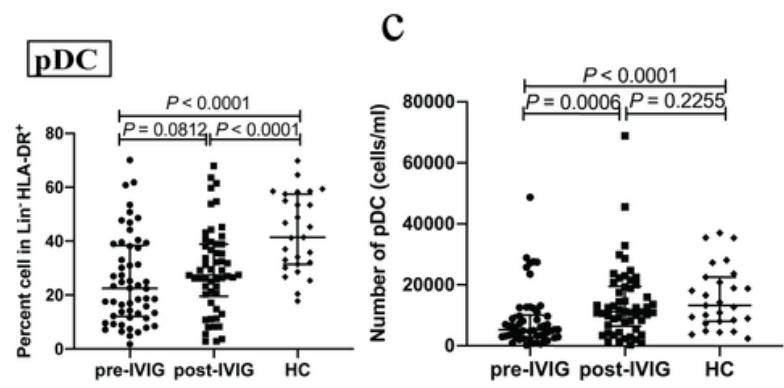

d $\mathrm{CD1c}^{+} \mathrm{mDC}$

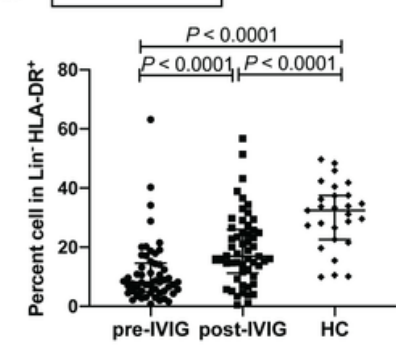

e

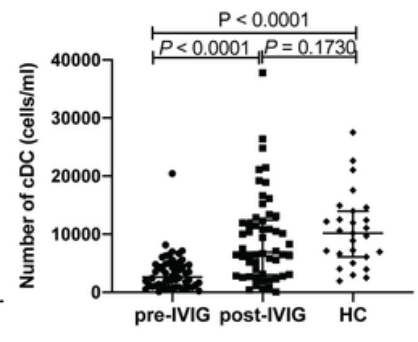

\section{Figure 1}

A profound reduction of circulating DC subsets in patients with KD. (a) Identification of circulating DC subsets in blood using flow cytometry, pan-DCs were gated as Lin- HLA-DR+, pDCs and CD1c+ mDCs were defined as Lin- HLA-DR+ CD123+ and Lin- HLA-DR+ CD1c+ cells, respectively. Representative profiles of the circulating DCs subsets are shown. (b-c) Plots show percentage and number of circulating pDCs in patients with KD $(n=54)$ and HCs $(n=27)$. (d-e) Plots show percentage and number of circulating CD1c+ mDCs in patients with KD $(n=54)$ and HCs $(n=27)$. Horizontal bars represent median values, and error bars represent the interquartile range. 
a
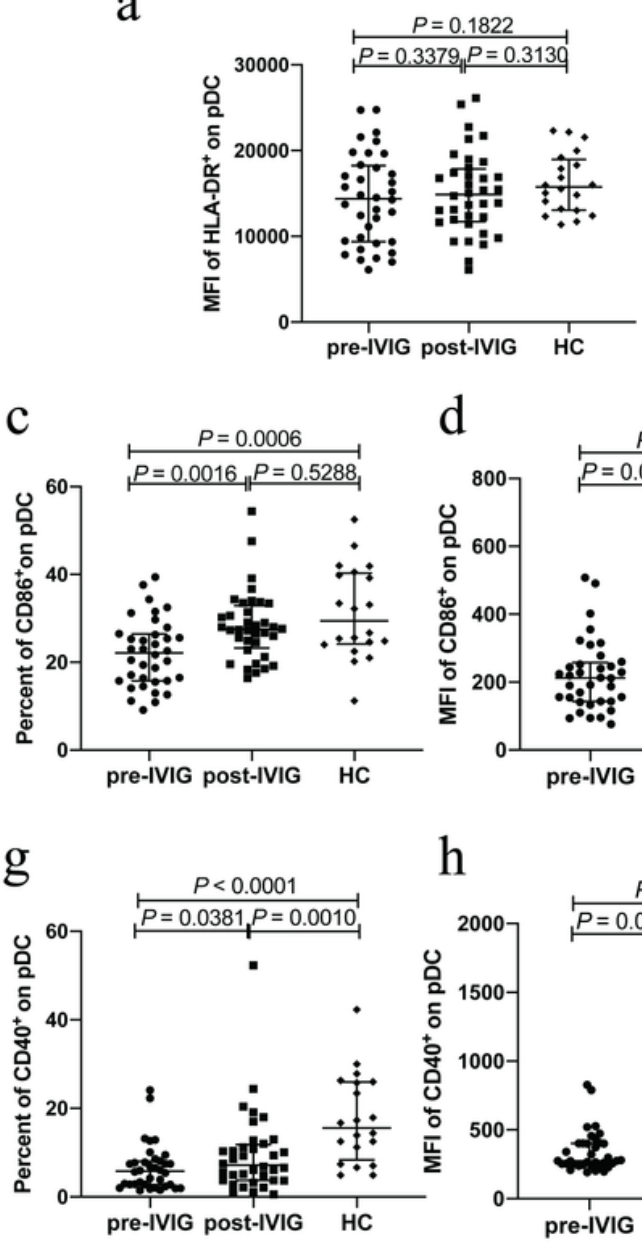

d

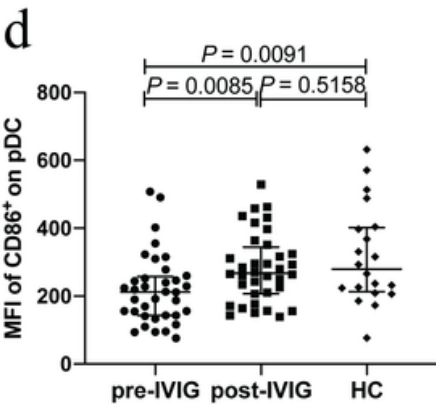

$\mathrm{h}$

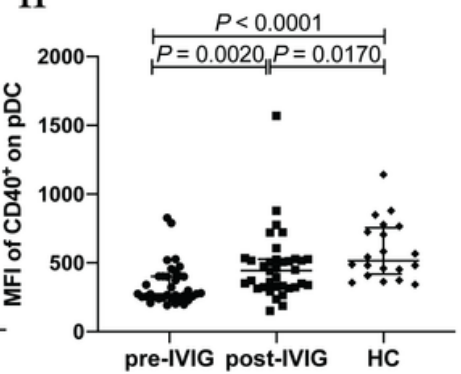

b

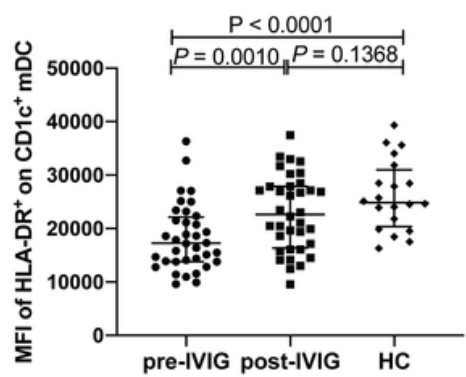

e

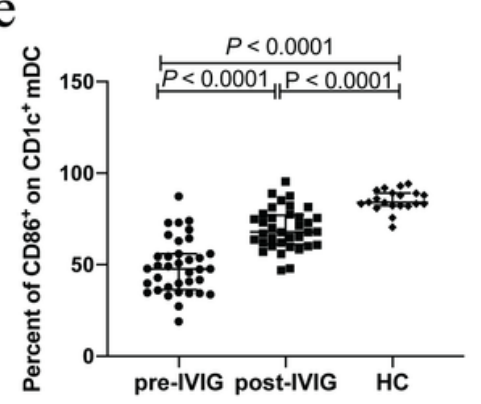

f

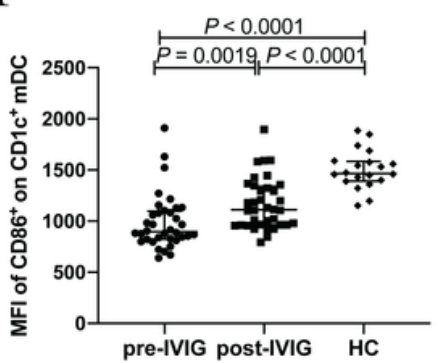

i

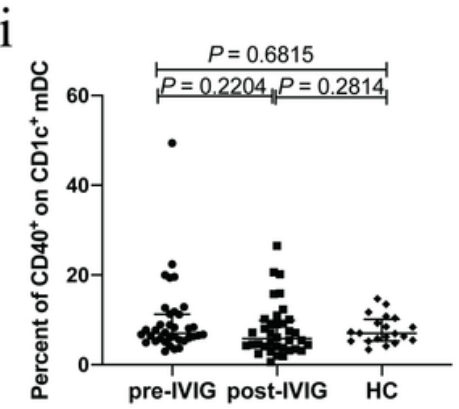

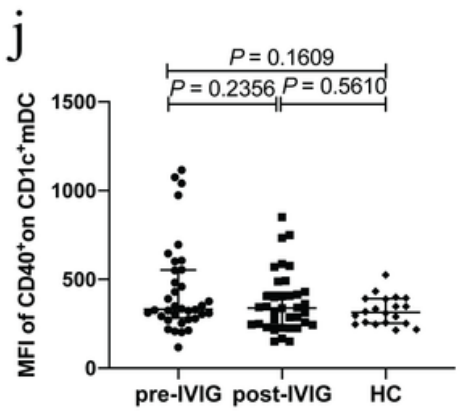

Figure 2

Flow cytometry analysis of the expression of HLA-DR, CD86 and CD40 on circulating DC subsets. Graphs show (a-b) HLA-DR MFI on pDCs and CD1c+ mDCs in patients with KD $(n=36)$ and $H C s(n=20)$. (c-d) Percentage and MFI of CD86+ on pDCs in patients with KD $(n=36)$ and HCs $(n=20)$. (e-f) Percentage and MFI of CD86+ on CD1c+ mDCs in patients with KD $(n=36)$ and HCs $(n=20)$. (g-h) Percentage and MFI of CD40+ on pDCs in patients with KD $(n=36)$ and HCs $(n=20)$. (i-j) Percentage and MFI of CD40+ on $\mathrm{CD} 1 \mathrm{c}+\mathrm{mDCs}$ in patients with $\mathrm{KD}(\mathrm{n}=36)$ and $\mathrm{HCs}(\mathrm{n}=20)$. Horizontal bars represent median values, and error bars represent the interquartile range. MFI, mean fluorescent intensity. 
$\mathrm{a}$

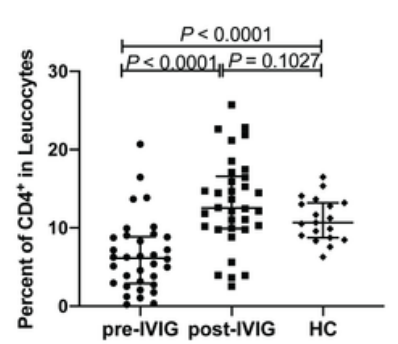

b

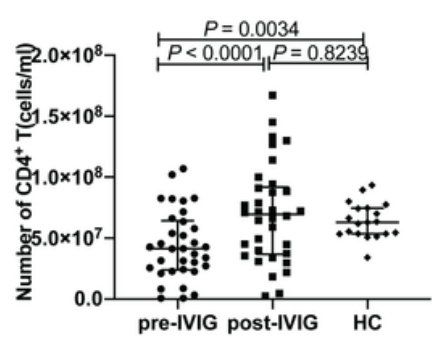

d

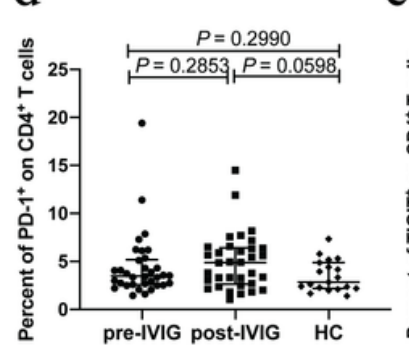

$\mathrm{e}$

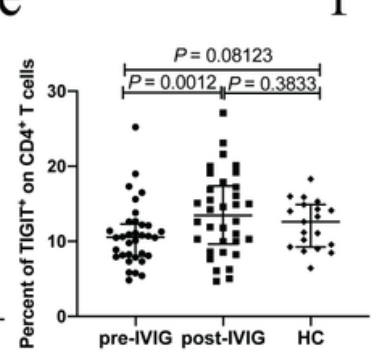

f

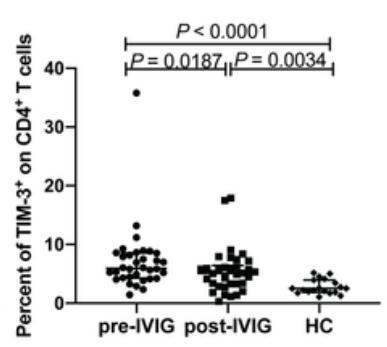

c
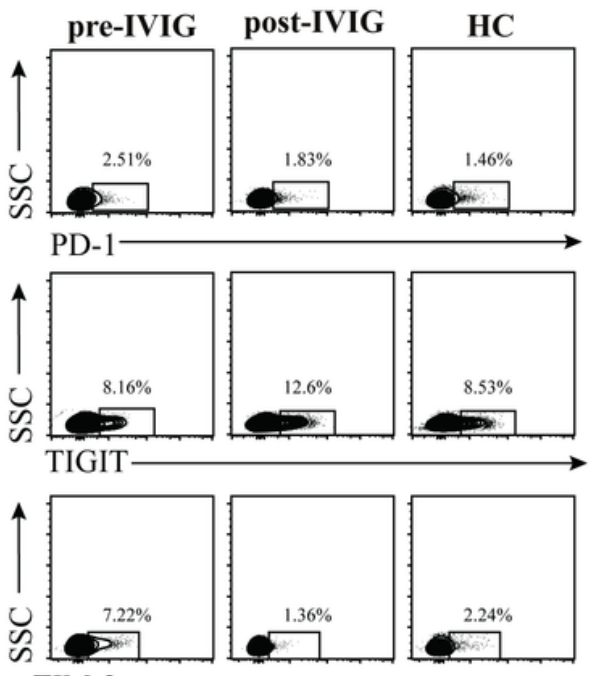

TIM-3

Figure 3

Flow cytometry detection of the expression of PD-1, TIGIT, and TIM-3 on CD4+ T cells. Graphs show (a-b) Percentage and number of CD4+ T cells among peripheral blood leucocytes in patients with KD $(n=34)$ and HCs $(n=19)$. (c) Representative dot plots of PD-1, TIGIT, and Tim-3 are shown on gated CD4+ T cells. (d) Expression of PD-1 on CD4+ T cells in patients with KD $(n=34)$ and HCs $(n=19)$. (e) Expression of TIGIT on CD4+ T cells in patients with KD $(n=34)$ and HCs $(n=19)$. (f) Expression of TIM-3 expression on CD4+ T cells in patients with KD $(n=34)$ and HCs $(n=19)$. Horizontal bars represent median values, and error bars represent the interquartile range. MFI, mean fluorescent intensity. 
a

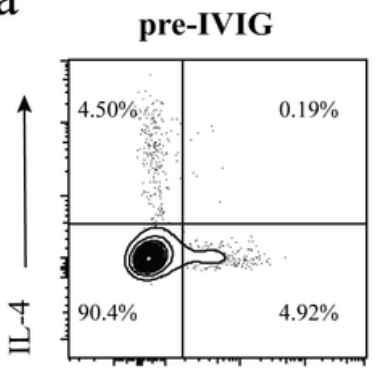

post-IVIG

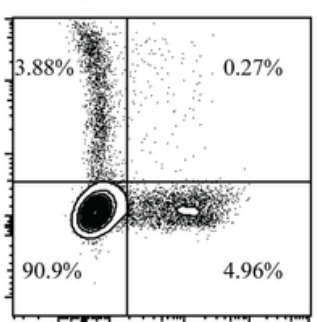

HC

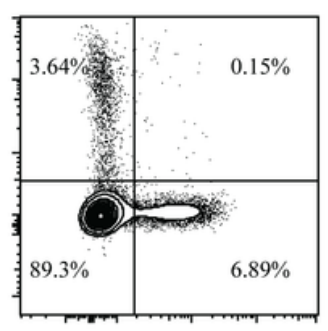

b

$\mathrm{C}$
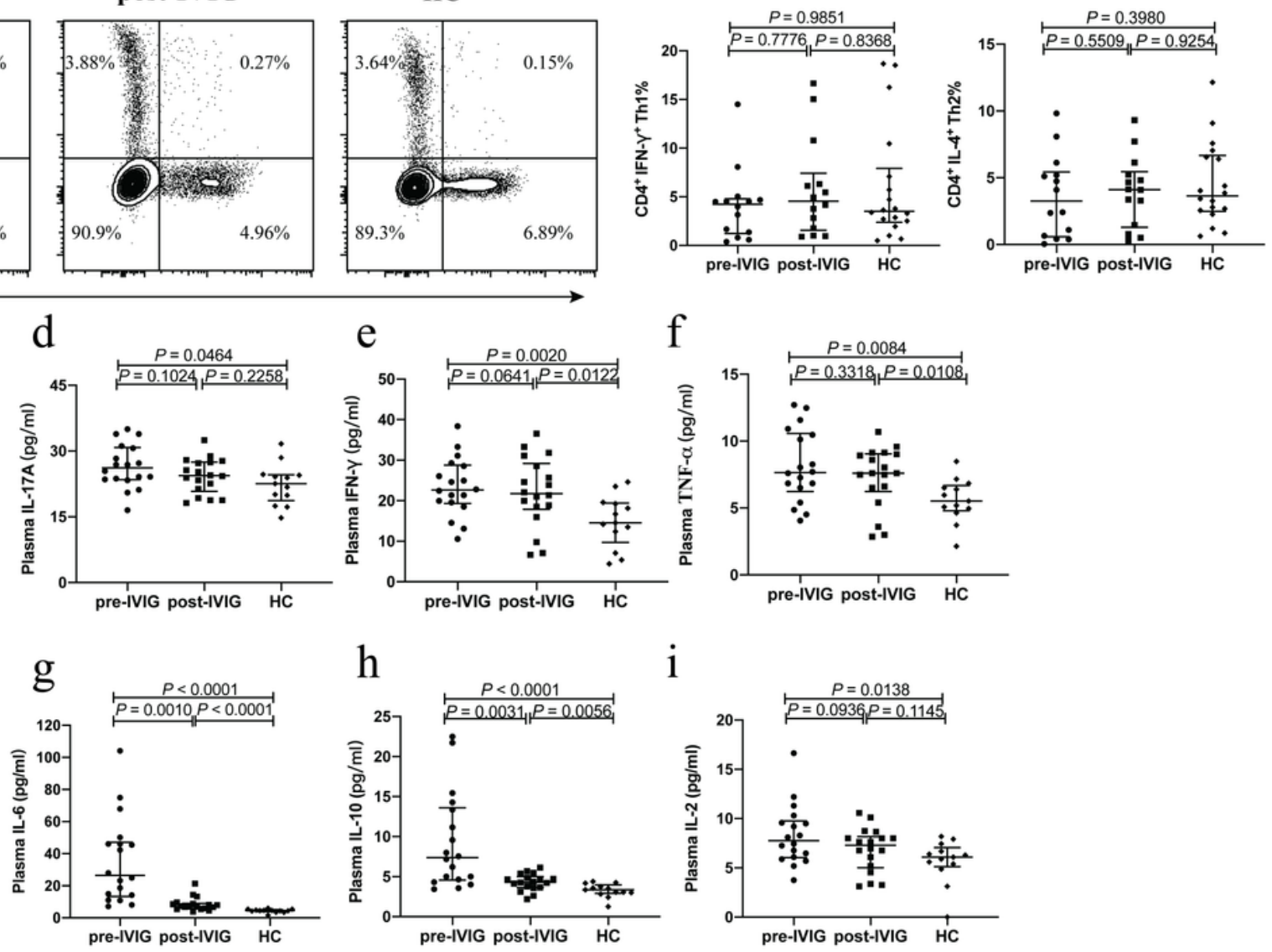

i

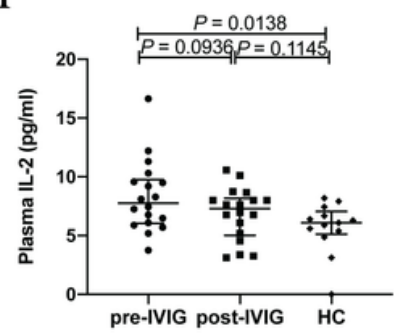

\section{Figure 4}

Flow cytometry analysis of the intracellular cytokine and plasma cytokine levels. (a) The gating strategy for determining CD4+ IFN- $\mathrm{y}+\mathrm{Th} 1$ and CD4+ IL-4+ Th2 cells is shown. (b) Percentage of circulating CD4+ IFN- $y+$ Th1 cells in patients with KD $(n=14)$ and HCs $(n=18)$. (c) Percentage of circulating CD4+ IL-4+ Th2 cells in patients with KD $(n=14)$ and HCs $(n=18)$. (d-i) Plasma levels of the cytokines IL-17A, IFN- $y$, TNF-a, IL-6, IL-10, IL-2 were detected in patients with KD ( $n=19)$ and HCs $(n=13)$ using cytometric bead array. Horizontal bars represent median values, and error bars represent the interquartile range. 